\title{
Hyperglycemia, Inflammatory Response and Infarct Size in Obstructive Acute Myocardial Infarction and MINOCA
}

Authors: Pasquale Paolisso MDe, Alberto Foà MD ${ }^{\mathrm{a}}$, Luca Bergamaschi MDª, Francesco Donati

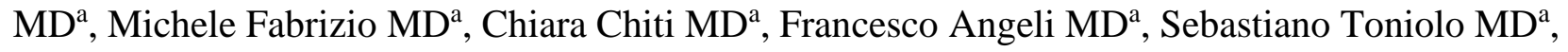
Andrea Stefanizzi MD, Matteo Armillotta MDa, Paola Rucci $\mathrm{PhD}^{\mathrm{b}}$, Gianni Casella $\mathrm{MD}^{\mathrm{c}}$, Gianmarco Iannopollo $\mathrm{MD}^{\mathrm{c}}$, Cinzia Marrozzini $\mathrm{MD}^{\mathrm{a}}$, Nazzareno Galiè MD, $\mathrm{PhD}^{\mathrm{a} *}$, Carmine Pizzi $\mathrm{MD}^{\mathrm{a} *}$.

\section{Affiliations:}

(a) Unit of Cardiology, Department of Experimental, Diagnostic and Specialty MedicineDIMES, University of Bologna, Italy.

(b) Division of Hygiene and Biostatistics, Department of Biomedical and Neuromotor Sciences, Alma Mater Studiorum, University of Bologna, Bologna, Italy.

(c) Unit of Cardiology, Maggiore Hospital, Bologna, Italy.

- The first two authors contributed equally to this work

*The last two authors contributed equally to this work

Corresponding author: Carmine Pizzi, FESC; Department of Experimental, Diagnostic and Specialty Medicine-DIMES (Padiglione 23), University of Bologna, Via Giuseppe Massarenti 9, 40138 Bologna, Italy. Tel. +39 051 6364933; Fax +39 051344859; e-mail carmine.pizzi@ unibo.it 


\begin{abstract}
Hyperglycemia has been associated with increased inflammatory indexes and larger infarct sizes in patients with obstructive acute myocardial infarction (obs-AMI). In contrast, no studies have explored these correlations in non-obstructive acute myocardial infarction (MINOCA). We investigated the relationship between hyperglycemia, inflammation and infarct size in a cohort of AMI patients that included MINOCA.

Patients with AMI undergoing coronary angiography between 2016 and 2020 were enrolled. The following inflammatory markers were evaluated: C-reactive protein, neutrophil-to-lymphocyte ratio (NLR), platelet-to-lymphocyte ratio (PLR) and neutrophil-to-platelet ratio (NPR). Myocardial infarct size was measured by peak high sensitivity troponin I (Hs-TnI) levels, left-ventricular-end-diastolicvolume (LVEDV) and left ventricular ejection fraction (LVEF).

The final study population consisted of 2450 patients with obs-AMI and 239 with MINOCA. Hyperglycemia was more prevalent among obs-AMI cases. In all hyperglycemic patients - obs-AMI and MINOCA - NLR, NPR, and LPR were markedly altered. Hyperglycemic obs-AMI subjects exhibited a higher Hs-TnI, a larger LVEDV and a lower LVEF compared to normoglycemic ones. Conversely, MINOCA patients showed similar myocardial damage, irrespective of glycemia.

Our data confirm the association of hyperglycemic obs-AMI with elevated inflammatory markers and larger infarct sizes. MINOCA patients exhibited modest myocardial damage, regardless of admission glucose levels.
\end{abstract}

Keywords: hyperglycemia, inflammation, infarct size, MINOCA, obstructive acute myocardial infarction. 


\section{Introduction}

Hyperglycemia frequently occurs in patients admitted for acute myocardial infarction (AMI), irrespective of a previously documented diabetes mellitus (DM) ${ }^{1}$. In particular, approximately $10 \%$ to $20 \%$ of non-diabetic AMI patients have significant hyperglycemia ${ }^{2}$. Recent data demonstrated that hyperglycemia is associated with an increased risk of major adverse cardiovascular events $(\mathrm{MACE})^{3,2}$. Additionally, amongst patients with large infarct sizes, hyperglycemia has been identified as a prognostic marker both in patients with and without diabetes ${ }^{4,5,6}$.

So far, it is unexplained whether elevated admission high glucose levels (aHGL) are a marker of more extensive myocardial damage or a prognostic risk factor in patients with $\mathrm{AMI}^{7}$.

In order to unravel the association between aHGL and the increased risk of adverse cardiovascular events, several potential explanations have been suggested. Systemic immune activation, modification of platelet function and thrombotic-fibrinolysis system, abnormal autonomic tone, increased oxidative stress, endothelial dysfunction and impaired myocardial contractility seem to play a role in myocardial damage $e^{8,9}$.

Etiopathogenetic mechanisms underlying hyperglycemia in the acute phase of myocardial infarction have not been fully elucidated. Blood glucose levels can be transiently elevated either as a stress response to acute illness (stress hyperglycemia), resulting from an inflammatory and adrenergic adaptation to ischemic injury (release of catecholamines and steroids and glycogenolysis induction), or as a reflection of an underlying abnormal glucometabolic state.

In the context of AMI, a series of ischemia-mediated pathophysiological events occur, generating an intense inflammatory response. Neutrophils are the first leukocytes detected in infarcted areas, followed by monocytes and lymphocytes, which, releasing proteo-enzymes and cytokines, phagocytize necrotic debris and promote the subsequent proliferative process ${ }^{10}$.

Additionally, activated platelets, besides acutely precipitating vascular obstruction, further amplify the inflammatory response interacting with neutrophils, monocytes and lymphocytes. Therefore, the 
role of inflammatory cells is not limited to the acute ischemic event but drives the chronic atherosclerotic process as well.

Recent accumulating evidence suggests that neutrophil-to-lymphocyte ratio (NLR), platelet-tolymphocyte ratio (PLR) and neutrophil-to-platelet ratio (NPR) might be considered as biomarkers of systemic inflammation and have been associated with poor clinical outcomes in various cardiovascular diseases, including acute coronary syndromes ${ }^{11,12,13,14}$.

The link between aHGL and inflammation is nowadays well established as it is the prognostic role of hyperglycemia in the context of AMI with obstructive coronary artery disease (obs-AMI). On the other hand, the relationship between hyperglycemia and inflammatory response in myocardial infarction with non-obstructive coronary arteries (MINOCA) is still poorly explored.

Our study sought to investigate the association between hyperglycemia and inflammatory status as well as myocardial damage/severity in patients with obs-AMI versus MINOCA. 


\section{Results}

A total of 2795 patients with suspected AMI who underwent coronary angiography within 72 hours of symptom onset were evaluated. Following diagnostic workup, a non-ischemic troponin elevation (e.g. sepsis, pulmonary embolism, myocarditis) was found in 94 patients, which were therefore excluded from the study. Among cases diagnosed with AMI, 12 patients (10 obs-AMI and 2 MINOCA) were excluded because blood glucose level at hospital admission was not available. The final study population consisted of 2450 patients with obs-AMI and 239 with MINOCA. Both groups were divided according to the presence of aHGL. Demographic and clinical characteristics are shown in Table 1. Overall, admission aHGL was noticed in 1017 patients (37.8\%), more frequently in patients with obs-AMI compared to MINOCA (40\% versus $16.7 \%$; $<<0.001$ ). The parameters of infarct size and myocardial damage/inflammation of each group are presented in Table 2 and Figure 2.

\section{Obstructive-AMI: hyperglycemic vs normoglycemic patients}

Over the 2450 patients with obs-AMI, hyperglycemia at admission was detected in 977 (40\%) while no cases of hypoglycemia were observed. Notably, among hyperglycemic patients, a known T2DM was recorded in approximately half cases while less than $10 \%$ of normoglycemic subjects were diabetic. Hyperglycemic patients exhibited a worse cardiovascular risk profile and more comorbidities compared to normoglycemic ones. In fact, they were older, generally overweight, with a higher prevalence of hypertension and a history of cardiovascular events. As expected, a hyperglycemic status reflected an underlying altered glycol-lipid profile and was associated with a greater comorbidity burden, such as atrial fibrillation and chronic lung disease. Over $90 \%$ of normoglycemic obs-AMI patients presented with typical angina, while the percentage dropped to $83 \%$ among hyperglycemic patients ( $\mathrm{p}$ 0.001). Lastly, STEMI diagnosis at admission was similar between subgroups. 


\section{MINOCA: hyperglycemic vs normoglycemic patients}

Among the 239 patients diagnosed with MINOCA, only $16.7 \%$ exhibited a hyperglycemic state at admission, and no cases of hypoglycemia were observed. Hyperglycemic patients were significantly older, with a higher prevalence of hypertension. Similarly to the obstructive cohort, hyperglycemic cases showed a worse metabolic profile, with higher cholesterol levels and a greater prevalence of T2DM. Interestingly, the glycemic status did not affect the history of cardiovascular events or the prevalence of comorbidities, except for atrial fibrillation which was more frequent among hyperglycemic patients. Again, typical angina was frequently observed among normoglycemic patients, while $35 \%$ of hyperglycemic subjects had a different clinical presentation $(p=0.004)$. STEMI was equally diagnosed among cohorts.

Impact of Admission Hyperglycemia on inflammatory markers and infarct size: obstructive-AMI vs MINOCA patients.

In obs-AMI patients, total white blood cell count, neutrophils, platelets, CRP and peak troponin T levels were significantly higher in aHGL group compared to normoglycemic cases (Table 2). Moreover, all inflammatory parameters (NLR, NPR and LPR) were markedly altered in hyperglycemic subjects, both at admission and after 24 hours (Table 2 and Table 3). Additionally, these patients exhibited a greater LVEDV and a lower LVEF compared to normoglycemic ones (Table 2). In the MINOCA cohort, inflammatory markers at admission - total white blood cell count, neutrophils, CRP, NLR, NPR and LPR - were significantly higher in aHGL group compared to normoglycemic patients while no differences were observed after 24 hours (Table 2). Importantly, hyperglycemic and normoglycemic subjects exhibited similar infarct sizes.

Comparing hyperglycemic obs-AMI and hyperglycemic MINOCA patients, similar values of inflammatory parameters were detected at admission. In contrast, higher levels of WBC and neutrophils were evident after 24 hours among the obs-AMI cohort. Notably, hyperglycemic obs- 
AMI subjects exhibited higher troponin levels, greater LVEDVs and a depressed LV function, all markers of larger infarct size. 


\section{Discussion}

Our study was focused on the interplay between hyperglycemia, inflammation and infarct size in a cohort of patients admitted with acute myocardial infarction, including cases of MINOCA, a still poorly investigated nosological entity.

Hyperglycemia was homogeneously associated with an increase of all inflammatory indices at admission, irrespective of the underlying ischemic pathophysiological mechanism, either obs-AMI or MINOCA. Importantly, hyperglycemia correlated with the detection of large infarct sizes only in patients with obs-AMI while no differences were observed between normoglycemic and hyperglycemic MINOCA cases.

\section{Hyperglycemia and inflammation markers in obstructive-AMI}

Among our overall study population, hyperglycemia was more frequently observed in patients with obs-AMI. This subgroup of hyperglycemic subjects exhibited an "inflammatory status" as expressed by increased levels of all measured inflammatory markers. High values of NLR, NPR, PLR and CRP had been previously described in this setting, and our results are in line with the existing literature, confirming the relationship between glycemic disorders and inflammation in the context of obs$\mathrm{AMI}^{21}$. Indeed, the activation of inflammatory mediators and pathways is vastly described as a cornerstone of atherosclerosis, not only in terms of chronic arterial remodelling but also favouring plaque instability and rupture ${ }^{22}$. Moreover, some studies have identified an association of elevated inflammatory markers, including NPR and NLR, with larger infarct sizes and an increased risk of short-term mortality ${ }^{12,11,23}$.

Hyperglycemia-mediated alterations may further precipitate the atherosclerotic process. In fact, hyperglycemia dysregulates endothelial homeostasis throughout several suggested mechanisms. Specifically, hyperglycemia causes an imbalanced production of reactive oxygen species (ROS) ${ }^{24,25}$, overexpresses adhesion molecules facilitating neutrophilic activation, stimulates the release of proinflammatory transcription factors and cytokines ${ }^{26}$. Importantly, not only does hyperglycemia 
amplify the inflammatory cascade, but it is also promoted by the inflammatory process itself throughout the generation of insulin-resistance and gluconeogenesis ${ }^{27,28,29}$. As a result, the interplay between hyperglycemia and inflammation triggers a vicious circle, ultimately leading to a heightened atherosclerotic burden with an increased mortality risk ${ }^{1,30}$.

\section{Hyperglycemia and inflammatory markers in MINOCA}

The main novelty of our study is that for the first time, we investigated the correlation between glycemic levels and previously described inflammatory markers in MINOCA patients. Similarly to the results observed in obs-AMI, hyperglycemic MINOCA subjects had higher values of NLR, NPR, and PLR than normoglycemic ones.

Shared underlying pathophysiological mechanisms may explain the complex interplay between hyperglycemia, inflammation and MINOCA. In particular, a central role seems to be played by endothelial dysfunction $^{31}$. In the MINOCA clinical setting, several studies have identified endothelial dysfunction as a determinant factor towards coronary artery vasoconstriction and vasospasm, resulting in myocardial ischemia ${ }^{32}$. As abovementioned, inflammation has the possibility of impairing endothelial function throughout the reduction of endothelium-derived vasodilators bioavailability, thereby decreasing the expression of endothelial nitric oxide synthase (eNOS) and nitric oxide synthesis. Another potential mechanism is the cytokine-mediated imbalance of the autonomic nervous system. Specifically, the hypothalamic-pituitary-adrenal axis response to inflammation causes an upregulation of the sympathetic system leading to coronary vasoconstriction, affecting both macro and micro-circulation ${ }^{33}$.

Although hyperglycemia in the context of MINOCA is still largely unexplored, it seems plausible that the same mechanisms described in obs-AMI may be valid in MINOCA as well. Supposedly, hyperglycemia can further precipitate the endothelial homeostasis and amplify the inflammatory process conferring an unbalanced vascular tone and a prothrombotic state, ultimately increasing the ischemic burden ${ }^{34,35}$. 


\section{Infarct size and hyperglycemia in Obstructive-AMI and MINOCA patients}

Hyperglycemic obs-AMI patients showed a larger infarct size than normoglycemic ones while glycemia did not affect the extent of myocardial damage among MINOCA cases. When comparing the two hyperglycemic study populations, obs-AMI subjets exhibited a more extensive myocardial injury as expressed by all the infarct markers evaluated: peak troponin, LVEDV and LVEF.

The link between hyperglycemia and large infarct size in the context of obs-AMI is well established, and our results are in line with previously published studies ${ }^{36}$. Over the past decades, multiple strategies have been adopted to assess the impact of admission hyperglycemia on the extent of myocardial damage, all leading toward the same direction. A study on 210 patients with STEMI showed a stronger correlation between peak troponin levels and infarct size measured by cardiac magnetic resonance among hyperglycemic subjects ${ }^{37}$. Similarly, in another STEMI cohort undergoing a single-photon emission computed tomography 5 days after admission, greater myocardial damage was observed in hyperglycemic cases $^{38}$. On the other hand, the impact of hyperglycemia on the extent of infarct size in MINOCA patients is still unexplored. Our study showed no differences between hyperglycemic vs normoglycemic patients and, overall, a modest infarct size, especially when compared to hyperglycemic obs-AMI subjects. The explanation to such results might be found in CMR studies focused specifically on the myocardial substrate of MINOCA cases. In particular, studies showed areas of myocardial oedema either associated with small necrotic regions with a typical patchy distribution or even without necrosis. It was interesting to observe that unlike obs-AMI, in MINOCA inflammatory parameters after 24 hours from hospitalization were similar in hyperglycemic and normoglycemic patients. While this data confirms the correlation between persistently elevated inflammatory markers and large infarct sizes - as proven by our hyperglycemic obs-AMI subgroup - questions arise regarding the importance of glycemia among the still hazy world of MINOCA. In fact, although inflammation and admission hyperglycemia are most probably inter- 
related in MINOCA as well, the prognostic role of glucose levels among such patients might be less relevant than in obs-AMI and require further focused investigations ${ }^{39}$.

\section{Study limitations}

Our study had several limitations. First, analyses were conducted on a relatively small sample size, especially regarding the MINOCA cohort. Second, not all laboratory parameters were available for each patient. Moreover, because of its cross-sectional design, the study does not allow to establish causal relationships between hyperglycemia, inflammatory markers and infarct size.

In patients with suspected DM, no definite rule-out criteria were adopted. However, not all patients can undergo an oral glucose tolerance test in the setting of AMI. Therefore, HbA1c could be a reasonable alternative in this clinical situation. 


\section{Materials and Methods}

\section{Patients}

All consecutive patients hospitalized for AMI (Policlinico Sant'Orsola-Malpighi Hospital and Maggiore Hospital, Bologna - Italy) who underwent coronary angiography (CAG) within the first 72 h from admission between January 2016 and March 2020 were included in the study. AMI was diagnosed in the presence of an increase and/or decrease of cardiac biomarker (troponin I high sensitivity - Tn I Hs) with at least one value above the 99th percentile upper reference limit associated with one of the following: symptoms of ischemia, new or presumed new significant ST-segment-T wave changes or new left bundle branch block, development of pathological Q waves in the EKG, and imaging evidence of new loss of viable myocardium or new regional wall motion abnormality ${ }^{15}$. MINOCA was diagnosed according to the 2016 ESC MINOCA Position Paper criteria ${ }^{16,17}$. Patients whose admission glycemia was not available were excluded from the study. Other exclusion criteria were severe valvular heart disease, prosthetic heart valves, severe anaemia, major acute bleeding, pulmonary embolism, fever $\left(38^{\circ} \mathrm{C}\right)$, hypertensive crisis, chronic renal failure (glomerular filtration rate $<30 \mathrm{~mL} / \mathrm{min} / 1.73 \mathrm{~m} 2$ ), autoimmune diseases, malignancies or ongoing cardiotoxic medications, and congenital heart disease.

Data were collected as part of an approved multicenter observational study called "AMIPE: Acute Myocardial Infarction, Prognostic and Therapeutic Evaluation" (ClinicalTrials.gov Identifier: NCT03883711). The present study was conducted according to the principles of the Declaration of Helsinki; all patients were informed about their participation in the registry and provided informed consent for the anonymous publication of scientific data.

\section{Data collection}

For each patient, demographic and baseline clinical data were collected, including sex, age, height, weight, body mass index (BMI), cardiovascular risk factors, family history of cardiovascular diseases. A standard 12-lead EKG was performed, and STEMI was diagnosed according to guidelines ${ }^{18}$. 
Glomerular filtration rate was calculated using the CKD-EPI formula. Blood samples were collected within 60 minutes from admission and after 24 hours. We also collected information on major epicardial coronary arteries obstruction, based on visual assessment during acute CAG performed by an expert interventional cardiologist.

\section{Inflammatory biomarkers and infarct size detection}

The inflammatory response was evaluated using the following parameters: NLR, NPR, PLR, CReactive Proteine. In particular, NLR is the ratio of neutrophil and lymphocyte counts, NPR is the ratio of neutrophil and platelet counts, and PLR is obtained by dividing the platelet count by the lymphocytes. The other laboratory parameters were determined according to standard protocols. Myocardial infarct size was measured by peak high sensitivity troponin I (Hs-TnI) levels within the first 24 hours of hospitalization. Comprehensive echocardiographic studies, including Doppler studies, were performed according to the current European recommendations ${ }^{19}$. Myocardial infarct size was also estimated using the left ventricular end-diastolic volume (LVEDV) and the left ventricular ejection fraction (LVEF).

\section{Blood Glucose and Definition of Hyperglycemia}

Blood glucose levels were assessed at admission as part of the standard evaluation. Pre-existing DM was defined as known DM at the time of hospitalization irrespective of the therapeutic management (either diet and lifestyle measures alone or additional administration of oral glucose-lowering medication and insulin) ${ }^{20}$. According to the American Heart Association Scientific Statement, patients were categorised based on admission glucose levels as follows: normoglycemia $<140 \mathrm{mg} / \mathrm{dl}$ and hyperglycemia $\geq 140 \mathrm{mg} / \mathrm{dl}^{2}$. 


\section{Statistical Analysis}

We analyzed the correlation of inflammatory and infarct size markers with hyperglycemia at hospital admission in patients with obs-AMI and in those with MINOCA. To this purpose, we first assessed the distribution of laboratory parameters using Shapiro-Wilks test and the homogeneity of variance using Levene's test. We then compared laboratory parameters and infarct sizes between patients with or without hyperglycemia using Mann-Whitney U test or Student's t-test as appropriate. Categorical variables were compared between groups using $\chi^{2}$ test. The significance level was set to $p<0.05$, and all analyses were performed using Stata 13.1 (Stata Corp., College Station, Texas, 2013) and IBM SPSS, version 25.0. 


\section{Conclusion}

In patients with acute myocardial infarction, hyperglycemia was associated with a larger infarct size in obs-AMI while no differences were observed in MINOCA. Hyperglycemic obs-AMI cases presented elevated inflammatory markers both ad admission and after 24 hours whereas in MINOCA this data was evident only at the time of hospitalization, paralleling the modest myocardial damage detected in such patients. Our findings have pathophysiological and therapeutic implications, especially for obs-AMI subjects who can benefit from aggressive secondary therapies. Further prospective studies are needed to assess the prognostic role of hyperglycemia in the heterogenous MINOCA entity. 
Authorship: all authors take responsibility for all aspects of the reliability and freedom from bias of the data presented and their discussed interpretation.

Conflict of interest: the authors declare that the research was conducted in the absence of any commercial or financial relationships that could be construed as a potential conflict of interest.

Author contributions: PP, LB and FD contributed conception and design of the study; FD, ST, MF, CC, FA, AS, GI and MA organised the database; LB and PR performed the statistical analysis; PP and $\mathrm{LB}$ wrote the first draft of the manuscript; $\mathrm{CP}$ and $\mathrm{AF}$ wrote sections of the manuscript. $\mathrm{CP}, \mathrm{AF}$, GC, CM and NG revised the article. All authors contributed to manuscript revision, read and approved the submitted version.

Acknowledgements: none

Sources of Funding: none

Disclosures: none 


\section{List of abbreviations:}

AMI: acute myocardial infarction

T2DM: Type 2 diabetes mellitus

MACE: major adverse cardiovascular events

aHGL: Admission High Glucose Levels

NLR: Neutrophil-to-lymphocyte ratio

PLR: Platelet-to-lymphocyte ratio

NPR: Neutrophil-to-platelet ratio,

MINOCA: Myocardial infarction with non-obstructive coronary arteries

Obs-AMI : obstructive myocardial infarction

CAG: coronary angiography

STEMI: ST-segment elevation acute myocardial infarction

LVEDV: left-ventricular-end-diastolic-volume

LVEF: left ventricular ejection fraction

HbA1C: glycosylated haemoglobin 


\section{References}

1. Capes SE, Hunt D, Malmberg K, Gerstein HC. Stress hyperglycaemia and increased risk of death after myocardial infarction in patients with and without diabetes: a systematic overview. Lancet 2000;355:773-778.

2. Deedwania P, Kosiborod M, Barrett E, Ceriello A, Isley W, Mazzone T, Raskin P. Hyperglycemia and Acute Coronary Syndrome: A Scientific Statement From the American Heart Association Diabetes Committee of the Council on Nutrition, Physical Activity, and Metabolism. Circulation 2008;117:1610-1619.

3. Shah B, Amoroso NS, Sedlis SP. Hyperglycemia in Nondiabetic Patients Presenting With Acute Myocardial Infarction. Am J Med Sci 2012;343:321-326.

4. Timmer JR, Hoekstra M, Nijsten MWN, Horst ICC van der, Ottervanger JP, Slingerland RJ, Dambrink J-HE, Bilo HJG, Zijlstra F, Hof AWJ van 't. Prognostic Value of Admission Glycosylated Hemoglobin and Glucose in Nondiabetic Patients With ST-Segment-Elevation Myocardial Infarction Treated With Percutaneous Coronary Intervention. Circulation 2011;124:704-711.

5. Mi S, Su G, Yang H, Zhou Y, Tian L, Zhang T, Tao H. Comparison of in-hospital glycemic variability and admission blood glucose in predicting short-term outcomes in non-diabetes patients with ST elevation myocardial infarction underwent percutaneous coronary intervention. Diabetol Metab Syndr 2017;9:20.

6. Khalfallah M, Abdelmageed R, Elgendy E, Hafez YM. Incidence, predictors and outcomes of stress hyperglycemia in patients with ST elevation myocardial infarction undergoing primary percutaneous coronary intervention. Diab Vasc Dis Res 2020;17(1):1479164119883983.

7. Singh K, Hibbert B, Singh B, Carson K, Premaratne M, Le May M, Chong A-Y, Arstall M, So D. Meta-analysis of admission hyperglycaemia in acute myocardial infarction patients treated with primary angioplasty: a cause or a marker of mortality? Eur Heart J Cardiovasc Pharmacother $2015 ; 1: 220-228$. 
8. Angeli F, Reboldi G, Poltronieri C, Lazzari L, Sordi M, Garofoli M, Bartolini C, Verdecchia P. Hyperglycemia in acute coronary syndromes: from mechanisms to prognostic implications. Ther Adv Cardiovasc Dis 2015;9:412-424.

9. Marik PE, Bellomo R. Stress hyperglycemia: an essential survival response!Crit Care. 2013;6:305.

10. Rock KL, Kono H. The Inflammatory Response to Cell Death. Annu Rev Pathol 2008;3:99-126.

11. Chen C, Cong BL, Wang M, Abdullah M, Wang XL, Zhang YH, Xu SJ, Cui L. Neutrophil to lymphocyte ratio as a predictor of myocardial damage and cardiac dysfunction in acute coronary syndrome patients. Integr Med Res 2018;7:192-199.

12. Somaschini A, Cornara S, Demarchi A, Mandurino-Mirizzi A, Fortuni F, Crimi G, Ferlini M, Camporotondo R, Gnecchi M, Visconti LO, De Ferrari GM. Neutrophil to platelet ratio: A novel prognostic biomarker in ST-elevation myocardial infarction patients undergoing primary percutaneous coronary intervention. Eur J Prev Cardiol 2019;16:2047487319894103.

13. Li W, Liu Q, Tang Y. Platelet to lymphocyte ratio in the prediction of adverse outcomes after acute coronary syndrome: a meta-analysis. Sci Rep 2017;7:40426.

14. Li H, Zhou Y, Ma Y, Han S, Zhou L. The prognostic value of the platelet-to-lymphocyte ratio in acute coronary syndrome: a systematic review and meta-analysis. Kardiol Pol. 2017; 75:666-673.

15. Thygesen K, Alpert JS, Jaffe AS, Chaitman BR, Bax JJ, Morrow DA, White HD, ESC Scientific Document Group, Thygesen K, Alpert JS, Jaffe AS, Chaitman BR, Bax JJ, Morrow DA, White HD, Mickley H, Crea F, Van de Werf F, Bucciarelli-Ducci C, Katus HA, Pinto FJ, Antman EM, Hamm CW, De Caterina R, Januzzi JL, Apple FS, Alonso Garcia MA, Underwood SR, Canty JM, Lyon AR, et al. Fourth universal definition of myocardial infarction (2018). Eur Heart J 2019;40:237-269.

16. Agewall S, Beltrame JF, Reynolds HR, Niessner A, Rosano G, Caforio ALP, De Caterina R, 
Zimarino M, Roffi M, Kjeldsen K, Atar D, Kaski JC, Sechtem U, Tornvall P. ESC working group position paper on myocardial infarction with non-obstructive coronary arteries. Eur Heart $J$ 2017;38:143-153.

17. Paolisso P, Bergamaschi L, Saturi G, D’Angelo EC, Magnani I, Toniolo S, Stefanizzi A, Rinaldi A, Bartoli L, Angeli F, Donati F, Rucci P, Mattioli AV, Taglieri N, Pizzi C, Galiè N. Secondary Prevention Medical Therapy and Outcomes in Patients With Myocardial Infarction With Non-Obstructive Coronary Artery Disease. Front Pharmacol 2020;10:1606.

18. Ibanez B, James S, Agewall S, Antunes MJ, Bucciarelli-Ducci C, Bueno H, Caforio ALP, Crea F, Goudevenos JA, Halvorsen S, Hindricks G, Kastrati A, Lenzen MJ, Prescott E, Roffi M, Valgimigli M, Varenhorst C, Vranckx P, Widimský P, ESC Scientific Document Group, Collet J-P, Kristensen SD, Aboyans V, Baumbach A, Bugiardini R, Coman IM, Delgado V, Fitzsimons D, Gaemperli O, Gershlick AH, et al. 2017 ESC Guidelines for the management of acute myocardial infarction in patients presenting with ST-segment elevation. Eur Heart J 2018;39:119-177.

19. Lang RM, Badano LP, Mor-Avi V, Afilalo J, Armstrong A, Ernande L, Flachskampf FA, Foster E, Goldstein SA, Kuznetsova T, Lancellotti P, Muraru D, Picard MH, Rietzschel ER, Rudski L, Spencer KT, Tsang W, Voigt J-U. Recommendations for Cardiac Chamber Quantification by Echocardiography in Adults: An Update from the American Society of Echocardiography and the European Association of Cardiovascular Imaging. Eur Heart J Cardiovasc Imaging 2015;16:233270.

20. American Diabetes Association. 2. Classification and Diagnosis of Diabetes: Standards of Medical Care in Diabetes-2019. Diabetes Care 2019;42:S13-S28.

21. Marfella R, Siniscalchi M, Esposito K, Sellitto A, Fanis U de, Romano C, Portoghese M, Siciliano S, Nappo F, Sasso FC, Mininni N, Cacciapuoti F, Lucivero G, Giunta R, Verza M, Giugliano D. Effects of Stress Hyperglycemia on Acute Myocardial Infarction: Role of inflammatory immune process in functional cardiac outcome. Diabetes Care 2003;26:3129-3135. 22. Galkina E, Ley K. Immune and Inflammatory Mechanisms of Atherosclerosis. Annu Rev 
Immunol 2009;27:165-197.

23. Centurión OA. Serum biomarkers and source of inflammation in acute coronary syndromes and percutaneous coronary interventions. Cardiovasc Revasc Med 2016;17:119-128.

24. Kitano D, Takayama T, Nagashima K, Akabane M, Okubo K, Hiro T, Hirayama A. A comparative study of time-specific oxidative stress after acute myocardial infarction in patients with and without diabetes mellitus. BMC Cardiovasc Disord 2016;16:102.

25. Giacco F, Brownlee M. Oxidative Stress and Diabetic Complications. Schmidt AM, ed. Circ Res 2010;107:1058-1070.

26. Lin B, Ginsberg MD, Busto R, Li L. Hyperglycemia triggers massive neutrophil deposition in brain following transient ischemia in rats. Neurosci Lett 2000;278:1-4.

27. Borst SE. The Role of TNF-alpha in insulin resistance. Endocrine 2004; 23:177-182.

28. Jager J, Grémeaux T, Cormont M, Le Marchand-Brustel Y, Tanti J-F. Interleukin-1 $\beta$ Induced Insulin Resistance in Adipocytes through Down-Regulation of Insulin Receptor Substrate1 Expression. Endocrinology 2007;148:241-251.

29. Barnes TM, Otero YF, Elliott AD, Locke AD, Malabanan CM, Coldren AG, Brissova M, Piston DW, McGuinness OP. Interleukin-6 amplifies glucagon secretion: coordinated control via the brain and pancreas. Am J Physiol Endocrinol Metab 2014;307:E896-905.

30. Mulder M de, Cornel J-H, Ploeg T van der, Boersma E, Umans VA. Elevated admission glucose is associated with increased long-term mortality in myocardial infarction patients, irrespective of the initially applied reperfusion strategy. Am Heart J 2010;160:412-419.

31. Tamis-Holland JE, Jneid H, Reynolds HR, Agewall S, Brilakis ES, Brown TM, Lerman A, Cushman M, Kumbhani DJ, Arslanian-Engoren C, Bolger AF, Beltrame JF, On behalf of the American Heart Association Interventional Cardiovascular Care Committee of the Council on Clinical Cardiology; Council on Cardiovascular and Stroke Nursing; Council on Epidemiology and Prevention; and Council on Quality of Care and Outcomes Research. Contemporary Diagnosis and Management of Patients With Myocardial Infarction in the Absence of Obstructive Coronary Artery 
Disease: A Scientific Statement From the American Heart Association. Circulation 2019;139:E891908.

32. Beijk MA, Vlastra WV, Delewi R, Hoef TP van de, Boekholdt SM, Sjauw KD, Piek JJ. Myocardial infarction with non-obstructive coronary arteries: a focus on vasospastic angina. Neth Heart J 2019;27:237-245.

33. Burford N, Webster N, Cruz-Topete D. Hypothalamic-Pituitary-Adrenal Axis Modulation of Glucocorticoids in the Cardiovascular System. Int J Mol Sci 2017;18:2150.

34. Sara JD, Taher R, Kolluri N, Vella A, Lerman LO, Lerman A. Coronary microvascular dysfunction is associated with poor glycemic control amongst female diabetics with chest pain and non-obstructive coronary artery disease. Cardiovasc Diabetol 2019;18:22.

35. Sardu C, Paolisso P, Sacra C, Mauro C, Minicucci F, Portoghese M, Rizzo MR, Barbieri M, Sasso FC, D’Onofrio N, Balestrieri ML, Calabrò P, Paolisso G, Marfella R. Effects of metformin therapy on COronary endothelial DYsfunction in prediabetic patients With stable angina and Non Obstructive Coronary Artery Stenosis: The CODYCE Multicenter Prospective Study. Diabetes Care 2019;42:1946-1955.

36. Eitel I, Hintze S, Waha S de, Fuernau G, Lurz P, Desch S, Schuler G, Thiele H. Prognostic Impact of Hyperglycemia in Nondiabetic and Diabetic Patients With ST-Elevation Myocardial Infarction: Insights From Contrast-Enhanced Magnetic Resonance Imaging. Circ Cardiovasc Imaging 2012;5:708-718.

37. Lonborg J, Vejlstrup N, Kelbaek H, Nepper-Christensen L, Jorgensen E, Helqvist S, Holmvang L, Saunamaki K, Botker HE, Kim WY, Clemmensen P, Treiman M, Engstrom T. Impact of Acute Hyperglycemia on Myocardial Infarct Size, Area at Risk, and Salvage in Patients With STEMI and the Association With Exenatide Treatment: Results From a Randomized Study.

Diabetes 2014;63:2474-2485.

38. Cruz-Gonzalez I, Chia S, Raffel OC, Sanchez-Ledesma M, Senatore F, Wackers FJ, Nathan DM, Jang I-K. Hyperglycemia on admission predicts larger infarct size in patients undergoing 
percutaneous coronary intervention for acute ST-segment elevation myocardial infarction. Diabetes Res Clin Pract 2010;88:97-102.

39. Pizzi C, Xhyheri B, Costa GM, Faustino M, Flacco ME, Gualano MR, Fragassi G, Grigioni

F, Manzoli L. Nonobstructive Versus Obstructive Coronary Artery Disease in Acute Coronary

Syndrome: A Meta-Analysis. J Am Heart Assoc 2016;5:E004185 


\section{Figure Legend}

Figure 1: Flow chart Study. CAG: coronary angiography; AMI: acute myocardial infarction; TpNOCA: troponin-positive non-obstructive coronary arteries; Obs-AMI: obstructive myocardial infarction; MINOCA: myocardial infarction with non-obstructive coronary arteries; aBGL: admission blood glucose level; aHGL: admission high glucose level; aNGL: admission normal glucose level.

Figure 2. Inflammatory markers in obstructive Acute Myocardial Infarction and Non-obstructive acute myocardial infarction. The blu colour denotes normoglycemic patients; the red colour represents hyperglycemic patients. AMI: Acute myocardial infarction, MINOCA: non-obstructive acute myocardial infarction, NLR: Neutrophil-to-lymphocyte ratio, NPR: Neutrophil-to-platelet ratio, PLR: Platelet-to-lymphocyte ratio. 
Table 1: Demographic, clinical, laboratory findings and treatment of obstructive-AMI and MINOCA patients, according to admission to hyperglycemia.

\begin{tabular}{|c|c|c|c|c|c|c|}
\hline & \multicolumn{2}{|c|}{$\begin{array}{l}\text { Obstructive-AMI } \\
\qquad \mathbf{N}=\mathbf{2 4 5 0}\end{array}$} & \multicolumn{4}{|c|}{$\begin{array}{c}\text { MINOCA } \\
\text { N }=\mathbf{2 3 9}\end{array}$} \\
\hline & aHGL & aNGL & $p$-value & aHGL & aNGL & $p$-value \\
\hline & $\mathrm{N}=977$ & $\mathrm{~N}=1473$ & & $\mathrm{~N}=40$ & $\mathrm{~N}=199$ & \\
\hline Age, years, median (IQR) & $72.0(62.0-80.0)$ & $68.0(58.0-78.0)$ & $<0.001$ & $74(67-81)$ & $68(53-77)$ & 0.001 \\
\hline Gender Female, n (\%) & $280(28.7)$ & $383(26)$ & 0.1 & $28(70)$ & $129(64.8)$ & 0.5 \\
\hline $\mathrm{BMI} \mathrm{Kg} / \mathrm{m}^{2}$, median (IQR) & $26.8(24.2-30.3)$ & $26.2(23.9-29.0)$ & 0.001 & $25.9(22.8-29.2)$ & $25.6(22.4-28.2)$ & 0.6 \\
\hline \multicolumn{7}{|l|}{ Cardiovascular risk factors } \\
\hline Current/past smoking, n (\%) & $547(56.3)$ & $908(62.4)$ & 0.007 & $13(32.5)$ & $88(44.7)$ & 0.1 \\
\hline Hypertension, n (\%) & $720(74.2)$ & $967(65.9)$ & $<0.001$ & $30(75)$ & $129(65.2)$ & 0.2 \\
\hline Dyslipidemia, n (\%) & $595(61.3)$ & $898(61.2)$ & 0.9 & $21(52.5)$ & $123(61.8)$ & 0.3 \\
\hline Type-2 diabetes, n (\%) & $477(48.8)$ & $113(7.7)$ & $<0.001$ & $12(30.0)$ & $11(5.5)$ & $<0.001$ \\
\hline \multicolumn{7}{|l|}{ Medical history } \\
\hline Previous AMI, n (\%) & $238(24.5)$ & $290(19.8)$ & 0.006 & $2(5.4)$ & $18(9.8)$ & 0.4 \\
\hline Previous stroke, n (\%) & $80(8.2)$ & $79(5.4)$ & 0.005 & $2(5.0)$ & $11(5.5)$ & 0.8 \\
\hline COPD, n (\%) & $122(12.5)$ & $152(10.3)$ & 0.09 & $5(12.5)$ & $21(10.6)$ & 0.7 \\
\hline PAD, n (\%) & $103(10.6)$ & $85(5.8)$ & $<0.001$ & $2(5)$ & $5(2.5)$ & 0.4 \\
\hline
\end{tabular}

Clinical Presentations

Obstructive-AMI

$\left(\frac{10}{2}\right.$

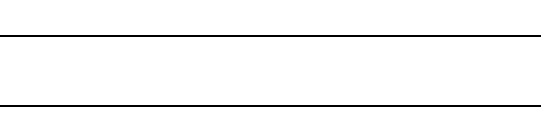




\begin{tabular}{|c|c|c|c|c|c|c|}
\hline Angina, $\mathrm{n}(\%)$ & $813(83.7)$ & $1337(91)$ & $<0.001$ & $28(70)$ & $170(85.4)$ & 0.02 \\
\hline HR, median (IQR) & $81(70-97)$ & $75(65-88)$ & $<0.001$ & $95(76-134)$ & $80(66-93)$ & $<0.001$ \\
\hline SBP, median (IQR) & $140(120-160)$ & $140(120-160)$ & 0.5 & $140(118-160)$ & $140(120-155)$ & 0.7 \\
\hline DBP, median (IQR) & $80(70-90)$ & $80(70-90)$ & 0.3 & $80(70-85)$ & $80(70-90)$ & 0.4 \\
\hline Atrial fibrillation, $\mathrm{n}(\%)$ & $103(10.6)$ & $93(6.4)$ & $<0.001$ & $13(32.5)$ & $14(7.1)$ & $<0.001$ \\
\hline STEMI, n (\%) & 468 (47.9) & $648(43.9)$ & 0.057 & $5(12.5)$ & $23(11.6)$ & 0.8 \\
\hline
\end{tabular}

\begin{tabular}{|c|c|c|c|c|c|c|}
\hline Laboratory Parameters & & & & & & \\
\hline Hemoglobin g/dL, median (IQR) & $13.6(12.1-15.0)$ & $14.0(12.7-15.1)$ & 0.001 & $13.2(12.1-14.8)$ & $13.4(12.1-14.5)$ & 0.9 \\
\hline Admission BGL level mg/dL, median (IQR) & $183(157-238)$ & $111(99-122)$ & $<0.001$ & $183(154-227)$ & $104(93-117)$ & $<0.001$ \\
\hline Discharge BGL level, mg/dl, median (IQR) & $114(97-145)$ & $98(85-112)$ & $<0.001$ & $105(92-127)$ & $97(85.0-111)$ & 0.02 \\
\hline HbA1c, mmol/mol, median (IQR) & $47(40-60)$ & $37(34-40)$ & $<0.001$ & $40(37-50)$ & $36(32-40)$ & 0.003 \\
\hline Creatinine mg/dl, median (IQR) & $1.0(0.9-1.3)$ & $0.9(0.8-1.1)$ & $<0.001$ & $1.0(0.7-1.2)$ & $0.8(0.7-1.0)$ & 0.04 \\
\hline C-TOT, mg/dL median (IQR) & $181(149-216)$ & $192(161-222)$ & $<0.001$ & $169(151-205)$ & $197(167-224)$ & 0.03 \\
\hline C-LDL, mg/dL median (IQR) & $111(85-139)$ & $121(93-149)$ & $<0.001$ & $97(84-127)$ & $118(97-144)$ & 0.04 \\
\hline Tryglicerides, median (IQR) & $116(84-165)$ & $112(83-153)$ & 0.02 & $116(89-143)$ & $111(80-153)$ & 0.8 \\
\hline \multicolumn{7}{|l|}{ Admission Medical Therapy } \\
\hline Aspirin, $\mathrm{n}(\%)$ & $374(38.6)$ & $501(34.2)$ & 0.03 & $8(20)$ & $50(25.1)$ & 0.5 \\
\hline P2Y12 Inhibitor,s n (\%) & $99(10.2)$ & $110(7.5)$ & 0.02 & $2(5)$ & $9(4.5)$ & 0.9 \\
\hline Beta-blockers, n (\%) & $401(41.4)$ & $520(35.6)$ & 0.004 & $17(42.5)$ & $60(30.2)$ & 0.1 \\
\hline RAAS inhibitors, $\mathrm{n}(\%)$ & $504(52)$ & $659(45.1)$ & 0.002 & $23(57.5)$ & $64(32.2)$ & 0.002 \\
\hline Statins, n (\%) & $297(30.7)$ & $395(27)$ & 0.048 & $15(37.5)$ & $51(25.6)$ & 0.1 \\
\hline
\end{tabular}




\begin{tabular}{|c|c|c|c|c|c|c|}
\hline \multicolumn{7}{|l|}{ Admission Glucose-lowering agents } \\
\hline Insulin sensitizers (metformin), $\mathrm{n}(\%)$ & $259(31.4)$ & $69(4.3)$ & $<0.001$ & $5(14.7)$ & $8(4)$ & 0.01 \\
\hline Insulin providers (sulfonylureas), n (\%) & $160(19.4)$ & $37(2.3)$ & $<0.001$ & $3(8.8)$ & $2(1.0)$ & 0.004 \\
\hline DPP-4 Inhibitors, n (\%) & $29(3.5)$ & $6(0.4)$ & $<0.001$ & $1(2.9)$ & $1(0.5)$ & 0.1 \\
\hline GLP-1 Agonist, n (\%) & $7(0.8)$ & $2(0.1)$ & 0.02 & 0 & 0 & 0.99 \\
\hline SGLT-2 Inhibitors, n (\%) & $3(0.4)$ & $2(0.1)$ & 0.06 & 0 & 0 & 0.99 \\
\hline Insulin, n (\%) & $120(14.6)$ & $27(1.7)$ & $<0.001$ & $2(5.9)$ & 0 & 0.001 \\
\hline
\end{tabular}

Continuous variables are presented as median (IQR) while categorical ones as $\mathrm{n}(\%)$. AMI = acute myocardial infarction; MINOCA = myocardial infarction with non-obstructive coronary arteries; Obs-AMI = obstructive acute myocardial infarction; $\mathrm{aHGL}=$ admission High Glucose Level; aNGL = admission normal glucose level; BMI = body max index; $\mathrm{COPD}=$ chronic obstructive pulmonary disease; $\mathrm{HR}=$ heart rate; $\mathrm{SBP}=$ systolic blood pressure; $\mathrm{DBP}=$ diastolic blood pressure; STEMI = ST-segment Elevation Myocardial Infarction; $\mathrm{BGL}=$ blood glucose level; HbA1c = glycated hemoglobin; C-TOT = total cholesterol; LDL-c = LDL cholesterol; RAAS = Renin-angiotensin-aldosterone system; DPP-4 = dipeptidyl peptidase 4; GLP-1 = glucagon-like peptide 1; SGLT-2 = sodium glucose co-transporter 2. 
Table 2: Inflammation markers and infarct size in Obstructive-AMI and MINOCA patients, according to admission hyperglycemia. The last column shows the comparison between hyperglycemic obstructive-AMI and MINOCA patients.

\begin{tabular}{|c|c|c|c|c|c|c|c|}
\hline & \multicolumn{2}{|c|}{$\begin{array}{l}\text { Obstructive-AMI } \\
\qquad \mathbf{N}=\mathbf{2 4 5 0}\end{array}$} & \multicolumn{4}{|c|}{$\begin{array}{c}\text { MINOCA } \\
\mathbf{N}=\mathbf{2 3 9}\end{array}$} & \multirow{2}{*}{ 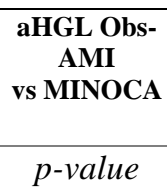 } \\
\hline & aHGL & aNGL & $p$-value & aHGL & aNGL & p-value & \\
\hline & $\mathrm{N}=977$ & $\mathrm{~N}=1473$ & & $\mathrm{~N}=40$ & $\mathrm{~N}=199$ & & \\
\hline \multicolumn{8}{|l|}{ Inflammation markers (admission - T0) } \\
\hline 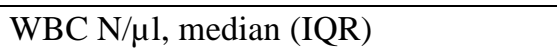 & $10.5(8.1-13.3)$ & $9.2(7.4-11.6)$ & $<0.001$ & $10.4(8.1-14.9)$ & $8.1(6.6-10.1)$ & $<0.001$ & ns \\
\hline Neutrophil N/ul, median (IQR) & $7197(5385-10249)$ & $6228(4678-8623)$ & $<0.001$ & $7933(5637-11443)$ & $5305(4053-7361)$ & $<0.001$ & ns \\
\hline 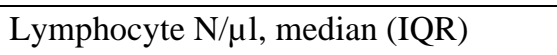 & $1710(1187-2530)$ & $1787(1312-2496)$ & 0.2 & $1736(1165-2164)$ & $1840(1385-2310)$ & 0.3 & ns \\
\hline PLTs count $\times 10^{9}$ per L, median (IQR) & $233(193-282)$ & $228(189-275)$ & 0.1 & $234(195-287)$ & $239(200-289)$ & 0.8 & ns \\
\hline CRP mg/dL, median (IQR) & $0.5(0.2-1.4)$ & $0.4(0.2-0.8)$ & $<0.001$ & $0.5(0.2-1.6)$ & $0.3(0.1-0.7)$ & 0.04 & ns \\
\hline \multicolumn{8}{|l|}{ Inflammation markers $(24 \mathrm{~h}-\mathrm{T1})$} \\
\hline 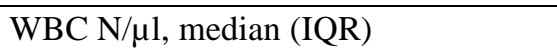 & $9.7(7.9-12.3)$ & $8.7(7.0-10.9)$ & $<0.001$ & $7.9(7.0-10.9)$ & $7.4(6.2-8.8)$ & 0.04 & 0.008 \\
\hline Neutrophil N/Ml, median (IQR) & $6935(5344-9392)$ & $5881(4425-7730)$ & $<0.001$ & $5505(3851-9122)$ & $4590(3519-6527)$ & 0.08 & 0.026 \\
\hline Lymphocyte $N / \mu 1$, median (IQR) & $1688(1187-2219)$ & $1839(1376-2407)$ & $<0.001$ & $2012(1042-2419)$ & $1817(1367-2304)$ & 0.9 & ns \\
\hline CRP mg/dL, median (IQR) & $1.1(0.4-4.3)$ & $0.7(0.3-1.8)$ & $<0.001$ & $1.0(0.4-2.3)$ & $0.5(0.2-1.0)$ & 0.06 & ns \\
\hline \multicolumn{8}{|l|}{ Infarct size } \\
\hline LVEDV ml, median (IQR) & $108(84-135)$ & $100(83-121)$ & 0.003 & $80(70-121)$ & $89(74-107)$ & 0.7 & 0.016 \\
\hline LV EF \%, median (IQR) & $47(40-56)$ & $55(45-60)$ & $<0.001$ & $59(50-61)$ & $60(53-62)$ & 0.8 & $<0.001$ \\
\hline Peak hs Troponin ng/L, median (IQR) & $6556(959-35531)$ & $2936(576-18164)$ & $<0.001$ & $369(133-901)$ & $461(113-1661)$ & 0.5 & $<0.001$ \\
\hline
\end{tabular}


Continuous variables are presented as median (IQR) while categorical ones as $\mathrm{n}(\%)$. AMI $=$ acute myocardial infarction; MINOCA $=$ myocardial infarction with non-obstructive coronary arteries; Obs-AMI = obstructive acute myocardial infarction; aHGL= admission High Glucose Level; aNGL = admission normal glucose level; WBC = White blood cell; PLTs = platelets; NLR = neutrophil-to-lymphocyte ratio; PLR = platelet-to-lymphocyte ratio; NPR = neutrophil-to-platelet ratio; CRP = C-reactive protein; LVEDV = left

ventricular end diastolic diameter; LVEDV, left ventricular end diastolic volume; LVEF = left ventricular ejection fraction; Hs = high sensitivity. 
Figure 1

AMIPE Registry

(Jan 2016 - March 2020)

$\mathrm{N}=3254$

No or $>72 \mathrm{~h} \mathrm{CAG}$ performed $\mathrm{N}=459$

No BGL

$\mathrm{N}=12$

Eligible patients $\mathrm{N}=2689$
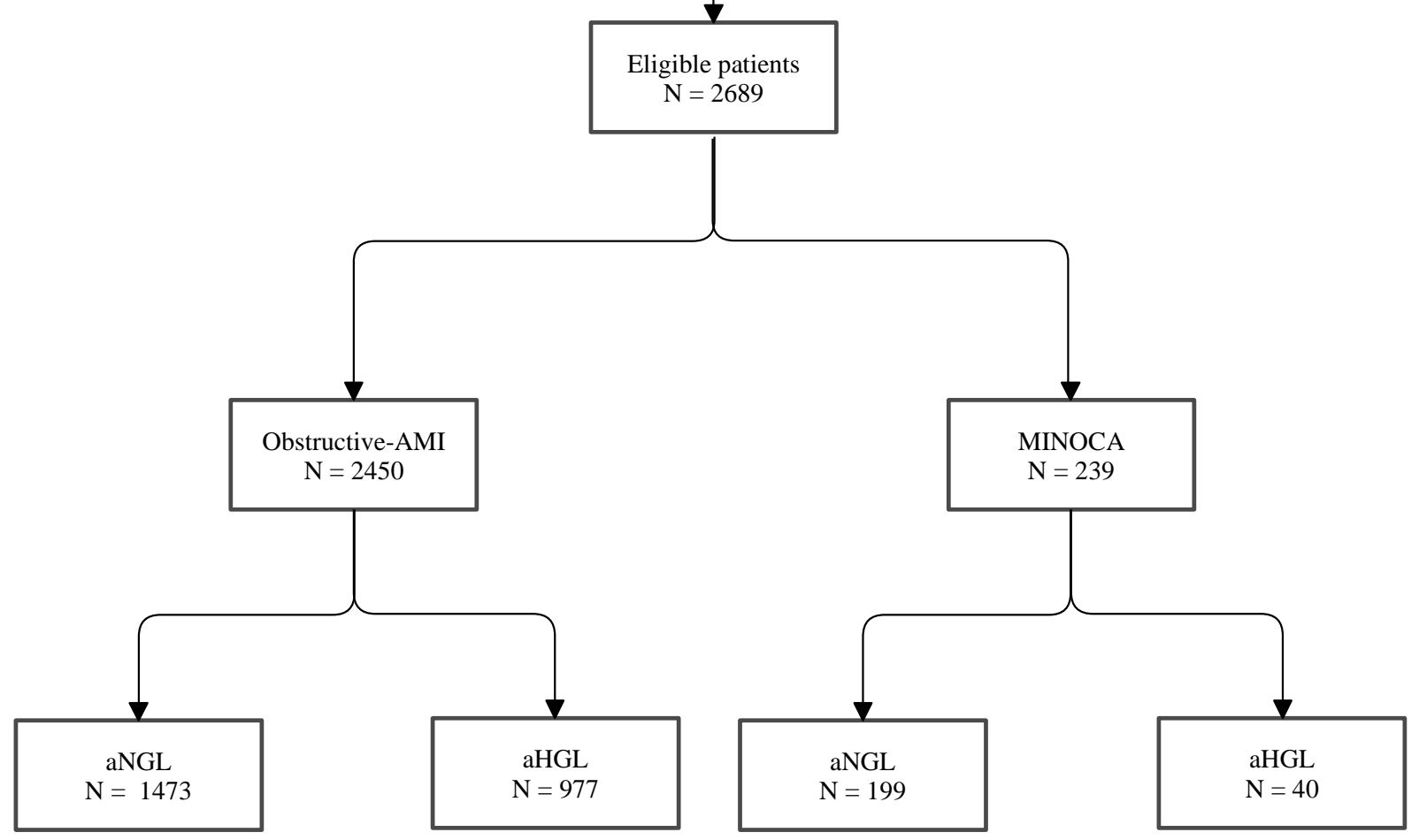

TpNOCA cardiac tra-cardiac $\mathrm{N}=94$

$$
\text { AMI }
$$

$\mathrm{N}=2701$
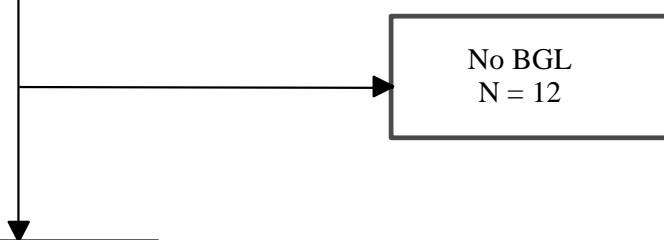
Figure 2

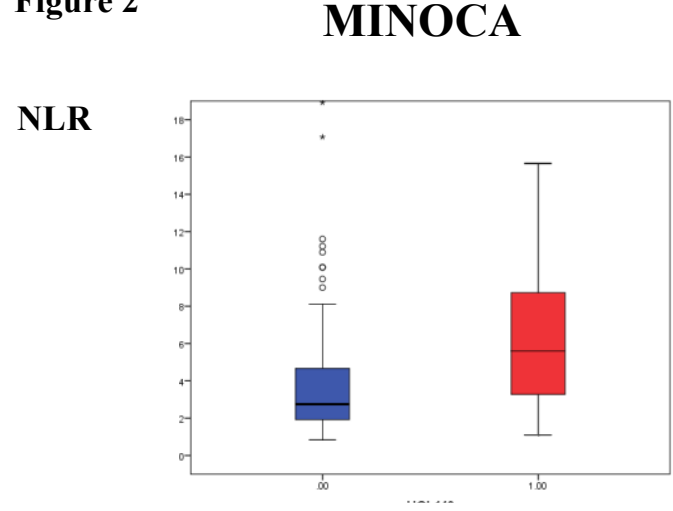

PLR

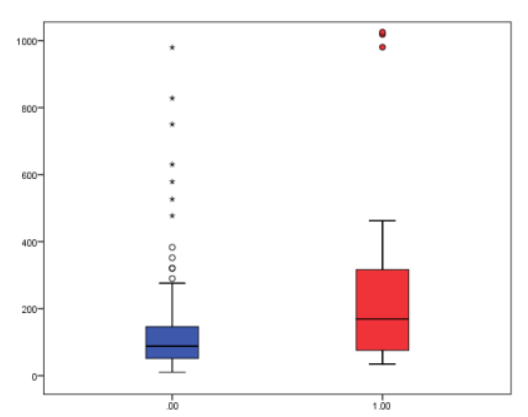

$P=0.002$

NPR

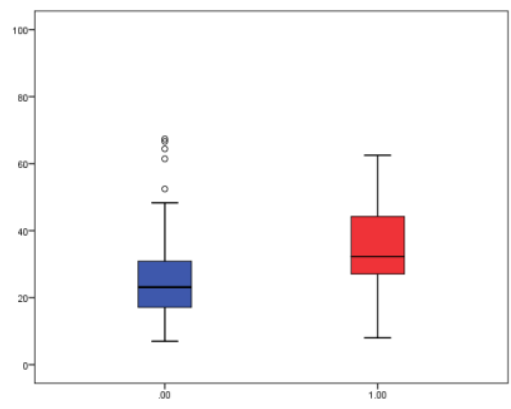

OBSTRUCTIVE-AMI

NLR

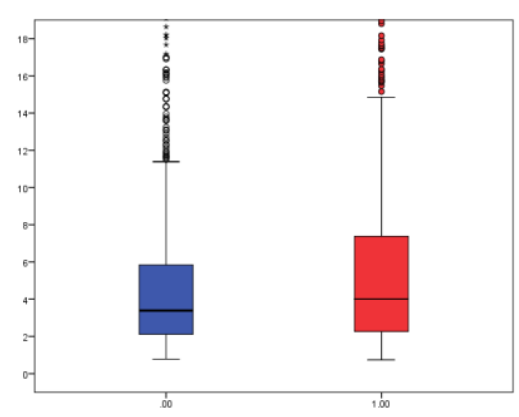

PLR

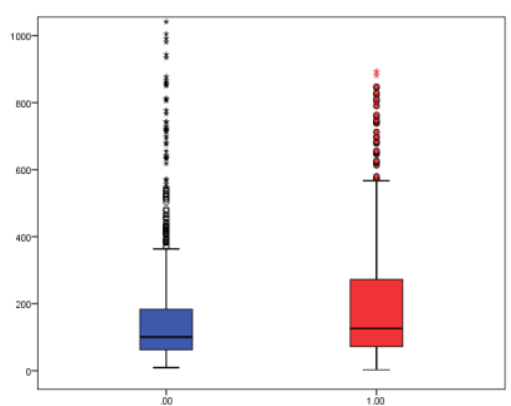

NPR

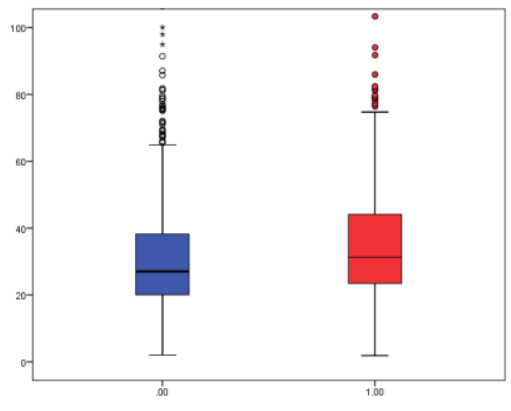

$\mathbf{P}<\mathbf{0 . 0 0 1}$

$\mathrm{P}<\mathbf{0 . 0 0 1}$

$\mathbf{P}<0.001$ 


\author{
Preprints (www.preprints.org) | NOT PEER-REVIEWED | Posted: 16 October $2020 \quad \underline{\text { doi:10.20944/preprints202010.0350.v1 }}$ \\ Preprints (www.preprints.org) | NOT PEER-REVIEWED | Posted: 16 October $2020 \quad$ doi:10.20944/preprints202010.0350.v1

dei:10.20944hreprints202010.0350v1

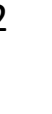

.

(1)

(1)

